\title{
Late onset Friedreich's disease: clinical features and mapping of mutation to the FRDA locus
}

\author{
G De Michele, A Filla, F Cavalcanti, L Di Maio, L Pianese, I Castaldo, O Calabrese, \\ A Monticelli, S Varrone, G Campanella, M Leone, M Pandolfo, S Cocozza
}

\begin{abstract}
Federico II
University, Naples, Italy

Department of milder than the "typical" form and that it maps to the same locus on chromosome 9.
\end{abstract}

Neurology

G De Michele

A Filla

F Cavalcant

L Di Maio

G Campanella

Department of

Molecular and

Cellular Biology and

Pathology and CEOS

(CNR)

L Pianese

I Castaldo

O Calabrese

A Monticelli

$S$ Varrone

$S$ Cocozza

Department of

Neurology, Ospedale

Maggiore, Novara,

Italy

$M$ Leone

Besta Neurological Institute, Milan, Italy

M Pandolfo

Correspondence to:

Dr Giuseppe De Michele,

Clinica Neurologica,

Università Federico II, via

Pansini 5, 80131 Napoli,

Italy.

Received 26 December

1993 and in revised form

26 January 1994.

Accepted 31 January 1994

(F Neurol Neurosurg Psychiatry 1994;57:977-979)

Friedreich's disease is an autosomal recessive disorder that represents the most common form of hereditary ataxia in large series. ${ }^{1}$ Age of onset was at about the time of puberty in the nine patients originally described by Friedreich, ${ }^{2}$ but a later onset has sometimes been described. For instance, Potts ${ }^{3}$ reported an onset between ages 27 and 36 years in three siblings. Geoffroy et $a l^{4}$ in an effort to define strict diagnostic criteria for Friedreich's disease, established that "true" Friedreich's disease must always begin before the end of puberty and at the latest before the age of 20. Harding ${ }^{1}$ considered the onset before 25 years of age an essential diagnostic criterion.

In 1989 we reported nine patients with Friedreich's disease phenotype but with age of onset ranging from 21 to 29 . We suggested that "late onset" Friedreich's disease may be a distinct genetic entity or may result from modifying secondary genes in some families. ${ }^{5}$

Chamberlain et al ${ }^{6}$ mapped the Friedreich's disease gene (FRDA) on the centromeric

\begin{abstract}
Twenty two patients from 17 families with Friedreich's disease phenotype but with onset ranging from the ages of 21 to 36 are described. Comparison with "typical" Friedreich's disease with onset before 20 years of age showed only a lower occurrence of skeletal deformities. The peripheral and central neurophysiological findings, sural nerve biopsy, and the neuroradiological picture did not allow the differentiation between "late onset" and "typical" Friedreich's disease. Duration of disease from onset to becoming confined to a wheelchair was five years longer in late onset patients. Sixteen patients and 25 healthy members from eight families were typed with the chromosome 9 markers MLS1, MS, and GS4 tightly linked to the FRDA locus. All families showed positive lod scores with a combined value of $5 \cdot 17$ at a recombination fraction of $\theta=0.00$. It is concluded that "late onset" Friedreich's disease is
\end{abstract}

region of chromosome 9, without evidence of genetic heterogeneity. The original findings have been confirmed in large studies on "typical" Friedreich's disease from European and North American populations. ${ }^{7}$ Klockgether et al, with markers of the FRDA region, found identical genotypes in three affected members of one family with late onset Friedreich's disease, but not in two unaffected siblings. ${ }^{8}$

Here we report the results of an expanded clinical and laboratory study and of a molecular genetic analysis in late onset Friedreich's disease. The aim of the study was to clarify whether it represents a distinct entity, clinically and genetically different from "typical" Friedreich's disease.

\section{Patients and methods}

At the Department of Neurology of the Federico II University of Naples, we saw 114 patients from southern and central Italy who fulfilled the following diagnostic criteria for Friedreich's disease: autosomal recessive inheritance or sporadic occurrence, progressive unremitting ataxia of limbs and gait, and absence of knee and ankle jerks. At least one of the following signs was present in the index cases: dysarthria, extensor plantar response, and echocardiographic evidence of hypertrophic cardiomyopathy. Ninety five of them had onset by 20 years of age and received the diagnosis of Friedrich's disease. The present study concerns the 19 patients whose onset age was older than 20 and three similar patients from northern Italy (referred by MP and $\mathrm{ML}$ ).

Severity of disease was scored according to the Inherited Ataxia Progression Scale (IAPS): stage 1, asymptomatic affected sibling; stage 2 , symptoms present but mild; stage 3, patient needs constant care and cannot work; stage 4, patient confined to a wheelchair.

The following microsatellite polymorphisms were studied for linkage analysis:GS4 (D9S111), MCT112/MS (D9S15), MLS1 (D9S202)..$^{9-12}$ For the DNA analysis a blood sample was taken in $50 \mathrm{mM}$ EDTA. Genomic DNAs were prepared according to described procedures. ${ }^{13}$ The polymerase chain reaction was used to detect microsatellite polymorphisms. DNA from each subject was amplified in $50 \mu \mathrm{l}$ of polymerase chain reaction buffer $(10 \mathrm{mM}$ Tris $\mathrm{HCl} \mathrm{pH} 8.3,1.5$ 
$\mathrm{mM} \mathrm{MgCl}, 50 \mathrm{mM} \mathrm{KCl}, 0.001 \%$ gelatine) containing $10 \mathrm{pmol}$ of ${ }^{32} \mathrm{P}$ labelled sense oligonucleotide primer, $10 \mathrm{pmol}$ of antisense oligonucleotide primer, and $10 \mathrm{nmol}$ of each deoxyribonucleotide triphosphate. One unit of Thermus aquaticus DNA polymerase (Perkin Elmer Cetus, Norwalk, CT, USA) was added and $100 \mu \mathrm{l}$ of mineral oil layered over the samples. Samples were then incubated at $95^{\circ} \mathrm{C}$, at annealing temperature $\left(57^{\circ} \mathrm{C}\right.$ for MLS1 and $60^{\circ} \mathrm{C}$ for GS4 and MS), and at $72^{\circ} \mathrm{C}$ for 45,50 , and 50 seconds respectively; this cycle was performed 36 times in a DNA-RNA amplifier (Biostar Violet, Rome, Italy).

The products of the polymerase chain reaction were loaded on denaturing $6 \%$ polyacrylammide gels, which were analysed by autoradiography after electrophoresis.

Pairwise lod scores were calculated with the Mlink program from the LINKAGE package version $5 \cdot 1^{14}$ between the markers and between the disease locus and the extended GS4-MS-MLS1 haplotype. A gene frequency of 0.006 was used.

\section{Results}

Eight patients with late onset Friedreich's disease were male and 14 were female $\left(\chi^{2}=\right.$ $1 \cdot 14$, NS). The patients were from 17 families, 12 from southern, three from northern, and two from central Italy. In two marriages parents were first cousins. A further seven affected siblings were not included in the study: four had onset before the age of 20 (at $4,5,6$, and 18 years), two were dead, and one was not available for examination. The segregation ratio, calculated according to Weinberg's "proband method", was 0.33 (95\% confidence interval $0 \cdot 18-0 \cdot 47$ ). Mean age of onset (SD) was $25.8(4 \cdot 2)$ (range 21-36) years, mean age at last observation was $38.9(7.8)$ (range 25-52) years, and mean duration of disease was 13.1 (8.2) (range 1-26) years. Eleven of our patients developed the disease after the age of 25 and two after the age of 30 . Eleven patients reproduced and had a total of 26 children.

The most frequent presenting symptom was gait ataxia $(91 \%)$, followed by lower limb weakness $(9 \%)$. Table 1 shows the percentage occurrence of clinical and laboratory findings in patients with late onset Friedreich's disease. Ten patients were in IAPS stage 2 (mean age (SD) $36.5(8.4)$ years), six in IAPS stage $3(38.8(7 \cdot 1)$ years), and six in IAPS stage $4(43 \cdot 2(6 \cdot 7)$ years $)$. They were compared with 22 "typical" patients with Friedreich's disease matched by sex and IAPS stage. Their mean age at onset was 11.4 (4.1) (range 1-19) years and disease duration 11.8 (8.5) (range 1-30) years. The only significant difference was a lower occurrence of skeletal deformities in late onset Friedreich's disease.

Peripheral nerve motor and sensory conduction studies were abnormal in all 16 patients with late onset Friedreich's disease examined and indicated a severe mainly sensory axonal degenerative neuropathy. Sural nerve biopsy in eight patients confirmed the
Table 1 Percentage occurrence of clinical and laboratory findings

\begin{tabular}{lcc}
\hline & $\begin{array}{c}L O F D \\
(n=22)\end{array}$ & $\begin{array}{c}F D \\
(n=22)\end{array}$ \\
\hline Gait and stance ataxia & 100 & 100 \\
Dysarthria & 82 & 86 \\
Nystagmus & 32 & 23 \\
Finger to nose dysmetria & 91 & 100 \\
Lower limb areflexia & 100 & 100 \\
Lower limb weakness & 50 & 73 \\
Lower limb wasting & 23 & 45 \\
Lower limb decreased vibration & & 86 \\
$\quad$ sense & 91 & 55 \\
Extensor plantar response & 45 & $91^{\star \star}$ \\
Pes cavus & 55 & $86^{\star}$ \\
Scoliosis & 55 & 14 \\
& 9 & 68 \\
Diabetes & 68 & 18 \\
Abnormal ECG & 14 & 46 \\
Abnormal EchoCG & 10 & \\
Abnormal CT & & \\
\hline
\end{tabular}

${ }^{\star} \mathrm{p}<0.05\left(\chi^{2}=3.93\right) ;{ }^{\star \star} \mathrm{p}<0.01\left(\chi^{2}=8.49\right)$.

TCT was performed on 10 patients with LOFD and 13 with FD. Patients with FD were matched by severity of disease and sex. FD = Friedreich's Disease; LOFD = late onset Friedreich's disease.

loss of large myelinated fibres, which was pronounced in five, with unimodal distribution of axon diameters. Motor and somatosensory evoked potentials were abnormal in the five investigated patients. Brainstem auditory evoked potentials were abnormal in five out of nine patients and visual evoked potentials were normal in all six evaluated patients.

CT showed vermian atrophy in one out of 10 patients. MRI (1-T field strength, Magnetom, Siemens AG, Germany) performed on five patients was abnormal in four, showing shrinkage of the cervical cord in all and atrophy of the vermis in two.

Disease progression through the IAPS stage was evaluated when personally observed or reliably referred. Ten patients reached IAPS stage 3 (loss of independent walking) after $10 \cdot 2(3 \cdot 1)$ (range $4-17$ ) years from the onset by the age of $34.9(5 \cdot 3)$ (range 25-44) and five patients reached IAPS stage 4 (wheelchair bound) after $17 \cdot 2(6 \cdot 5)(12-27)$ years by the age of $40.6(5.4)$ (range 35-49). The interval from onset to IAPS stage 3 was $8.2(3.7)$ years in 50 patients with Friedreich's disease and that from onset to IAPS stage 4 was $12 \cdot 1(4 \cdot 7)$ years in 39 . The last interval was significantly different between late onset Friedreich's disease and Friedreich's disease (Wilcoxon rank sum test, $\mathrm{p}=0.04)$.

Linkage analysis was performed for 16 patients and 25 healthy members from eight families. The homogeneity test, by HOMOG program (version 3.3), showed no heterogeneity. No recombination was found between markers. Table 2 shows the results of linkage analysis between the extended MLS1-MS-GS4 haplotype and the disease locus. The peak lod score value was $5 \cdot 17$ at a recombination fraction $\theta=0.00$, showing the absence of any recombination event.

The frequency of the alleles of the various markers used was comparable with that found in "typical" patients with Friedreich's disease and the normal population. No common MLS1-MS-GS4 haplotype was found in late onset Friedreich's disease families (data not shown). 
Table 2 Pairwise lod scores between late onset Friedreich's disease locus and extended MLS1-MS-GS4 haplotype

\begin{tabular}{llllll}
\hline \multicolumn{5}{l}{ Recombination fraction } \\
\cline { 2 - 6 } Family & 0.00 & 0.05 & 0.10 & 0.20 & 0.30 \\
\hline A $^{\star}$ & $\mathbf{1 . 0 5}$ & 0.91 & 0.77 & 0.49 & 0.25 \\
B & 0.96 & 0.84 & 0.73 & 0.48 & 0.25 \\
C & 1.45 & 1.32 & 1.17 & 0.85 & 0.49 \\
D & 0.60 & 0.51 & 0.43 & 0.27 & 0.13 \\
E & 0.60 & 0.51 & 0.43 & 0.27 & 0.13 \\
F & 0.25 & 0.17 & 0.10 & 0.02 & 0.00 \\
G & 0.12 & 0.12 & 0.11 & 0.08 & 0.04 \\
Total & 0.12 & 0.09 & 0.06 & 0.02 & 0.00 \\
\hline
\end{tabular}

${ }^{\star}$ The pedigree of family $\mathrm{A}$ has been published. ${ }^{17}$

\section{Discussion}

We found an age of onset older than 20 in 19 out of 114 patients $(17 \%)$ with a Friedreich's disease phenotype. A comparison of their clinical and laboratory findings with those of patients with Friedreich's disease of typical age of onset showed only a lower occurrence of pes cavus and scoliosis. This finding may be explained by an onset after full skeletal development. The occurrence of echocardiographic abnormalities was similar in patients with late onset Friedreich's disease (14\%) and matched patients with Friedreich's disease $(18 \%)$, but lower than that found in our study on a population of 50 patients with Friedreich's disease (34\%). ${ }^{15}$ The occurrence of abnormalities on CT was lower but not significantly in patients with late onset Friedreich's disease. The neurophysiological and morphological study of the peripheral nerve did not differentiate late onset Friedreich's disease from Friedreich's disease. The occurrence of abnormal motor and somatosensory evoked potentials and brain auditory evoked potentials was similar to that reported in Friedreich's disease. Visually evoked potentials were always normal in late onset Friedreich's disease, whereas they were abnormal in $69 \%$ of "typical" patients with Friedreich's disease. ${ }^{16}$

Disease progression, as indicated by years from onset to becoming confined to a wheelchair, was slower in patients with late onset Friedreich's disease. Late onset Friedreich's disease seems to be less severe than "typical" Friedreich's disease, not only because of a later onset, but also because of a milder course. Many of our patients are still ambulant without support at advanced ages (up to 52 years), and many married and had children. The finding that a later onset could determine a slower progression may be relevant from a prognostic point of view.

Previous data suggested that late onset Friedreich's disease might be due to secondary modifying genes acting on FRDA or to a different mutation. ${ }^{5}$ The mapping of the FRDA gene on chromosome 9 allowed a molecular genetic analysis in patients with late onset Friedreich's disease to test locus heterogeneity. Our results clearly showed that Friedreich's disease and late onset Friedreich's disease map to the same locus.

Our study cannot answer the question whether the same mutation causes Friedreich's disease and late onset Friedreich's disease or if these diseases are caused by two or more allelic mutations. The coexistence of patients with onset before and after 20 years of age in some families shows that the same genetic defect can cause both Friedreich's disease and late onset Friedreich's disease. ${ }^{17}$ Environmental factors or secondary modifying genes might account for the differences in age onset and progression. A different allelic mutation may, however, still be present in the families where all the affected have a late onset. The absence of a common haplotype in patients with late onset Friedreich's disease suggests that, in any case, there is not a common "late onset" mutation in these pedigrees.

Strict diagnostic criteria were required for the molecular genetic studies that led to the FRDA mapping. Gene identification will probably expand the Friedreich's disease phenotype proving also that some atypical cases may be caused by the same mutation. We believe, however, that age of onset before 20 years should not be considered an essential diagnostic criterion. Late onset is possible in Friedreich's disease and it occurs in a substantial number of patients; the clinical picture is not distinguishable from that of early onset cases, but the progression of the disease is slower.

This study was partially supported by grants from CNR (No 91.04180 ) and from Italian Telethon (to MP).

1 Harding AE. Friedreich's ataxia: a clinical and genetic study of 90 families with an analysis of early diagnostic criteria and intrafamilial

2 Friedreich N. Über Ataxie mit besonderer Berucksichtigung der hereditären Formen. Virchows Arch Pathol Anat Histol 1876;68:145-245.

3 Potts WJ. A case with Friedreich's disease with onset late in life. Brain 1906;29:405-6.

4 Geoffroy G, Barbeau A, Breton A, et al. Clinical description and roentgenologic evaluation of patients with Friedreich's ataxia. Can $\mathcal{f}$ Neurol Sci 1976;3:279-86.

5 De Michele G, Filla A, Barbieri F, et al. Late onset recessive ataxia with Friedreich's disease phenotype. $\mathcal{f}$ Neurol Neurosurg Psychiatry 1989;52:1398-401.

6 Chamberlain S, Shaw J, Rowland A, et al. Mapping of mutation causing Friedreich's ataxia to human chromosome 9. Nature 1988;334:248-50.

7 Chamberlain S, Shaw J, Wallis J, et al. Genetic homogeneity at the Friedreich's ataxia locus on chromosome 9. Am $\mathcal{F}$ Hum Genet 1989;44:518-21.

8 Klockgether T, Chamberlain S, Wüllner U, et al. Late onset Friedreich's ataxia. Molecular genetics, clinical neurophysiology and magnetic resonance imaging. Arch Neurol 1993;50:803-6.

9 Sirugo G, Keats B, Fujita R, et al. Friedreich ataxia in Lousiana Acadians: demonstration of a founder effect by analysis of microsatellite-generated expanded haplotypes. Am f Hum Genet 1992;50:559-66.

10 Fujita R, Hanauer A, Sirugo G, Heilig R, Mandel JL Additional polymorphisms at marker loci D9S5 and Additional polymorphis D9S15 generate extended haplotypes in linkage disequilibrium with Friedre

11 Wallis J, Williamson R, Chamberlain S. Identification of hypervariable microsatellite polymorphism within
D $9 S 15$ tightly linked to the Friedreich's ataxia locus. D9S15 tightly linked to the

12 Pandolfo M, Munaro M, Cocozza S, et al. A dinucleotide repeat polymorphism (MLS1-D9S202) in the Friedreich's ataxia region of chromosome $9 \mathrm{q} 13-\mathrm{q} 21 \cdot 1$ Hum Mol Genet 1993;2:822.

13 Maniatis T, Fritsch EF, Sambrook J. Molecular cloning. A laboratory manual.Cold Spring Harbor: Cold Spring Harbor Laboratory, 1982

14 Lathrop GM, Lalouel JM, Julier C, Ott J. Multilocus linkage analysis in humans: detection of linkage and estimation of recombination. Am 7 Hum Genet 1985;37: 482-98.

15 Filla A, De Michele G, Caruso G, Marconi R, Campanella G. Genetic data and natural history of Friedreich's disease: a study of 80 Italian patients. f Neurol 1990;237.345-51.

16 Caruso G, Santoro L, Perretti A, et al. Friedreich's ataxia electrophysiologic and histologic findings in patients electrophysiologic and histologic findings
and relatives. Muscle Nerve 1987;10:503-15.

17 Filla A, De Michele G, Cavalcanti F, Santorelli F, Santoro L, Campanella G. Intrafamilial phenotype variation in Friedreich's disease: possible exceptions to diagnostic criteria. $\mathcal{F}$ Neurol $1991 ; 238: 147-50$. 